\title{
Mackendrick: A Maple Package Oriented to Symbolic Computational Epidemiology
}

\author{
Juan Ospina ${ }^{1}$ and Doracelly Hincapie ${ }^{2}$ \\ ${ }^{1}$ EAFIT University \\ School of Sciences and Humanities \\ Department of Basic Sciences \\ Division of Physical Engineering \\ Logic and Computation Group \\ Medellin, Colombia \\ judoan@epm.net.co \\ ${ }^{2}$ University of Antioquia \\ National School of Public Health \\ Department of Basic Sciences \\ Group of Epidemiology \\ Medellin, Colombia \\ doracely@guajiros.udea.edu.co
}

\begin{abstract}
A Maple Package named Mackendrick is presented. Such package is oriented to symbolic computational epidemiology.
\end{abstract}

\section{Introduction}

We present here, the maple package Mackendrick which we have constructed for the solution of certain problems in symbolic computational epidemiology. Our package does not incorporate any kind of element of artificial intelligence, but for some of the problems that we solved, will be very funny to have some computer algebra system with artificial intelligence. The problems that we can solve here are linear problems but such problems only can be solved using computer algebra, due the involved calculations are very tedious and long as to be implemented by hand using pen and paper only.

Our emblematic problems are situations of spatial propagation of directly transmitted diseases when boundary conditions are involved at the form of endemic boundaries from where the disease spreads towards the interior of the habitat. More over, we consider here the extra complication that arises from the inclusion of the effects of heterogeneity of contact between individuals.

A fundamental epidemiological magnitude is the well know basic reproductive rate, denoted $R_{0}$. The principal function of the our package Mackendrick is the computation of the explicit analytical form of the $R_{0}$ for certain spatial models of disease diffusion with heterogeneity effects. We need here, computer algebra, because that it is required is a symbolic expression for $R_{0}$ and not a number or a graphic. Due, our package is constructed under maple platform, then our package has numerical and graphical computational power too. 
The package is loaded with

restart:

with (Mackendrick);

and the notification is

[dielou, difumemoestra, memo, memoyf, mysol, prosize,sir, veneco, venecomemo];

which is the list of procedures that are contained within Mackendrick.

In the following sections, the commands of Mackendrick are presented.

\section{The Command mysol}

For example, the procedure mysol solves the following problem:

$$
\begin{array}{r}
\frac{\partial}{\partial t} u(r, t)-\frac{\delta_{1}\left(\frac{\partial}{\partial r} u(r, t)+r \frac{\partial^{2}}{\partial r^{2}} u(r, t)\right)}{r} \\
-\delta_{2} \int_{0}^{t} \frac{M_{0}(t-\tau)\left(\frac{\partial}{\partial r} u(r, \tau)+r \frac{\partial^{2}}{\partial r^{2}} u(r, \tau)\right)}{r} d \tau \\
-\left(\beta_{1} S_{0}-\gamma_{1}\right) u(r, t)-\beta_{2} S_{0} \int_{0}^{t} u(r, \tau) M_{1}(t-\tau) d \tau+ \\
\gamma_{2} \int_{0}^{t} u(r, \tau) M_{2}(t-\tau) d \tau=0
\end{array}
$$

with the boundary condition

$$
u(a, t)=\mu_{b} e^{-\eta t}
$$

The procedure mysol needs as inputs the specifical forms of the functions $M_{0}(t)$, $M_{1}(t)$ and $M_{2}(t)$. Here we present two cases.

\subsection{Without Memory}

For example when

$$
M_{0}(t)=0, M_{1}(t)=0, M_{2}(t)=0,
$$

and with the instructions

M0 $:=0:$ M1 $:=0:$ M2: $=0:$

mysol (M0, M1, M2);

Mackendrick produces the following solution [1] 
$u(r, t)=\frac{\mu_{b} e^{-\eta t} J_{0}(\sqrt{\lambda(-\eta)} r)}{J_{0}(\sqrt{\lambda(-\eta)} a)}+\sum_{i=1}^{1} \sum_{n=1}^{\infty} \frac{-2 e^{S_{i, n} t} \mu_{b} J_{0}\left(\frac{\alpha_{n} r}{a}\right) \alpha_{n}}{\left(S_{i, n}+\eta\right)\left(J_{1}\left(\alpha_{n}\right)\right) a^{2}\left(\frac{d}{d S_{i, n}} \lambda\left(S_{i, n}\right)\right)}$.

The corresponding basic reproductive rate is given by

$$
R_{0}=\frac{\beta_{1} S_{0} a^{2}}{\gamma_{1} a^{2}+\alpha_{n}^{2} \delta_{1}} .
$$

The function $\lambda(s)$ at (4) has the form

$$
\lambda(s)=-\frac{s-\beta_{1} S_{0}+\gamma_{1}}{\delta_{1}},
$$

and the parameters denoted $S_{i, n}$ at (4) are the solutions of the equation on $s$

$$
-\frac{s-\beta_{1} S_{0}+\gamma_{1}}{\delta_{1}}=\frac{\alpha_{n}^{2}}{a^{2}}
$$

where $\alpha_{n}$ are the zeroes of the Bessel function $J_{0}(x)$ [2].

\subsection{With Exponential Memory}

$$
M_{0}(t)=e^{-\epsilon_{0} t}, M_{1}(t)=e^{-\epsilon_{1} t}, M_{2}(t)=e^{-\epsilon_{2} t},
$$

and with instructions

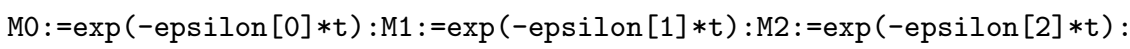

mysol (M0, M1, M2);

Mackendrick produces the following solution

$$
u(r, t)=\frac{\mu_{b} e^{-\eta t} J_{0}(\sqrt{\lambda(-\eta)} r)}{J_{0}(\sqrt{\lambda(-\eta)} a)}+\sum_{i=1}^{4} \sum_{n=1}^{\infty} \frac{-2 e^{S_{i, n} t} \mu_{b} J_{0}\left(\frac{\alpha_{n} r}{a}\right) \alpha_{n}}{\left(S_{i, n}+\eta\right)\left(J_{1}\left(\alpha_{n}\right)\right) a^{2}\left(\frac{d}{d S_{i, n}} \lambda\left(S_{i, n}\right)\right)} .
$$

The corresponding basic reproductive rate is given by

$$
R_{0}=\frac{S_{0} a^{2} \epsilon_{2} \epsilon_{0}\left(\beta_{1} \epsilon_{1}+\beta_{2}\right)}{\epsilon_{1}\left(\alpha_{n}^{2} \delta_{1} \epsilon_{0} \epsilon_{2}+\alpha_{n}^{2} \delta_{2} \epsilon_{2}+\gamma_{2} a^{2} \epsilon_{0}+\gamma_{1} a^{2} \epsilon_{2} \epsilon_{0}\right)} .
$$

The function $\lambda(s)$ at (9) has the form

$$
\lambda(s)=\left(s-\beta_{1} S_{0}+\gamma_{1}-\frac{\beta_{2} S_{0}}{s+\epsilon_{1}}+\frac{\gamma_{2}}{s+\epsilon_{2}}\right)\left(-\delta_{1}-\frac{\delta_{2}}{s+\epsilon_{0}}\right)^{-1},
$$

and the parameters denoted $S_{i, n}$ at (9) are the solutions of the equation on $s$

$$
\sqrt{\left(s-\beta_{1} S_{0}+\gamma_{1}-\frac{\beta_{2} S_{0}}{s+\epsilon_{1}}+\frac{\gamma_{2}}{s+\epsilon_{2}}\right)\left(-\delta_{1}-\frac{\delta_{2}}{s+\epsilon_{0}}\right)^{-1}}=\frac{\alpha_{n}}{a} .
$$




\section{The Command veneco}

The procedure veneco solves the following problem

$$
\frac{d}{d t} X_{i}(t)-\frac{\beta n X_{i}(t)}{k}+\gamma X_{i}(t)-\frac{2 \beta n\left(\sum_{j=1}^{k} \nu X_{j}(t)-\nu X_{i}(t)\right)}{k}=0 .
$$

with the instruction

$\operatorname{veneco}(n)$;

our Mackendrick gives the following form of the basic reproductive rate 3]

$$
R_{0, k}=\frac{\beta n(1+2 \nu k-2 \nu)}{\gamma k}
$$

\section{Conclusions}

We believe that the Maple package Mackendrick can be useful within the domain of symbolic computational epidemiology. Our Mackendrick can solve certain complex spatial epidemic models. The method of solution that Mackendrick incorporates is the Laplace transform technique with the application of the Bromwich integral and residue theorem for the realization of the inverse Laplace transform [4]. Also, Mackendrick involves certain theorem of Linear Algebra, which is presented in 3. This theorem must be introduced ad hoc but it is possible that with the future development of artificial intelligence, such theorem can be proved directly by the computer algebra system that is the background of Mackendrick. We hope that at the future our package can be extended and applied to more numerous and complex problems in mathematical epidemiology.

\section{References}

1. Hincapie, D., Ospina, J.: Basic reproductive rate of a spatial epidemic model using computer algebra software. In Valafar, F., Valafar, H., eds.: Proceedings of the 2005 International Conference on Mathematics and Engineering Techniques in Medicine and Biological Sciences. (2005)

2. Bowman, F.: Introduction to Bessel Functions. Dover Publications Inc., New York (1958)

3. Rodriguez, D.J., Torres-Sorando, L.: Models of infectious diseases in spatially heterogeneous environments. Bulletin of Mathematical Biology 63 (2001) 547-571

4. Apostol, T.M.: Mathematical Analysis. Addison-Wesley Publishing Company, Reading, Massachusetts (1988) 